Proceedings of the International School and Conference on Optics and Optical Materials, ISCOM07, Belgrade, Serbia, September 3-7, 2007

\title{
Possibility of Enhancement of Amplitude-Squared Squeezing in Mixing with Coherent Light Beam Using a Mach-Zehnder Interferometer
}

\author{
D.K. MishrA* \\ Physics Department, University of Allahabad, Allahabad-211002, India \\ and \\ V.S. Mehta College of Science, Bharwari, Kaushambi-212201, U.P., India \\ Possibility of enhancement of amplitude-squared squeezing for Mach- \\ Zehnder interferometer is investigated and it is found that the maximum \\ amount of amplitude-squared squeezing obtained by the present mixing is \\ not greater than that obtained by Prakash and Mishra.
}

PACS numbers: 42.50 Dv

\section{Introduction}

Sudarshan and Glauber [1, 2], independently, showed that the density operator of radiation can be written as

$$
\hat{\rho}=\int \mathrm{d}^{2} \alpha P(\alpha)|\alpha\rangle\langle\alpha|
$$

where $\alpha \equiv \alpha_{\mathrm{r}}+\mathrm{i} \alpha_{\mathrm{i}}$ is a complex number, $\mathrm{d}^{2} \alpha \equiv \mathrm{d} \alpha_{\mathrm{r}} \mathrm{d} \alpha_{\mathrm{i}}$ and $|\alpha\rangle$ is the eigenstate of the annihilation operator $\hat{a}(\hat{a}|\alpha\rangle=\alpha|\alpha\rangle)$, carets $\left(^{\wedge}\right)$ denote operators, and is called a coherent state [1]. Radiation is said to be in a classical state if the weight function $P(\alpha)$ is not more singular than Dirac delta function $\delta^{2}(\alpha)$ or is non-negative whereas non-classical features do not follow such a condition. Earlier, the non-classical features, antibunching [3, 4] and squeezing [5-7], were studied with academic interest [3-6, 8], as they provide instances where classical physics fails and only a quantum theory can explain the facts. In squeezed optical field, the fluctuations in one quadrature component are smaller than those associated with a coherent state and it has potential application in reduction of

*e-mail: kndmishra@rediffmail.com 
noise level in optical communication [8-10] and in detection of the extremely weak gravitational radiation [11-13]. Hong and Mandel [14, 15] generalized the concept of ordinary squeezing to the $2 N$-th-order case and Hillery $[16,17]$ proposed the so-called amplitude-squared squeezing (ASS) which is generated in some nonlinear interactions such as second harmonic generation [16], degenerate parametric amplification [17], two-photon absorption [17], anharmonic oscillator [18], JaynesCummings model [19] etc. The generalization of ASS was carried to orders $>2$ and one finds it in literatures [20].

Recently, Prakash and Mishra [21] studied ASS (which can be detected in principle by homodyning method [22]) in a light beam having a Gaussian statistics and reported that this non-classical feature can be enhanced in a simple linear mixing with a classical light beam via a beam splitter. Parametric amplification $[23,24]$ is one such process, which generates such Gaussian light beams that exhibit non-classical features. We investigate here the possibility of enhancement of amplitude-squared squeezing for Mach-Zehnder interferometer as a next order generalization. It is found that, under some conditions, amplitude-squared squeezing at the output of second beam splitter is enhanced as compared to that at one of output of the first beam splitter. But the maximum amount of amplitude-squared squeezing obtained by the present mixing is not greater than that obtained by Prakash and Mishra [21].

\section{Amplitude-squared squeezing in input Gaussian light beam and output beams in Mach-Zehnder interferometer}

If we consider hermitian operators $\hat{Y}_{1,2}$ defined by $\hat{Y}_{1}+\mathrm{i} \hat{Y}_{2}=\hat{a}^{2}$, i.e., $\hat{Y}_{1}=$ $\frac{1}{2}\left(\hat{a}^{\dagger 2}+\hat{a}^{2}\right)$ and $\hat{Y}_{2}=\frac{i}{2}\left(\hat{a}^{\dagger 2}-\hat{a}^{2}\right)$, the non-classical feature called ASS [16, 17] is defined by $\left\langle\left(\Delta \hat{Y}_{1}\right)^{2}\right\rangle<\left\langle\hat{a}^{\dagger} \hat{a}+\frac{1}{2}\right\rangle$ or $\left\langle\left(\Delta \hat{Y}_{2}\right)^{2}\right\rangle<\left\langle\hat{a}^{\dagger} \hat{a}+\frac{1}{2}\right\rangle$. We can also consider the more general operator, $\hat{Y}_{\theta} \equiv \frac{1}{2}\left(\hat{a}^{\dagger 2} \mathrm{e}^{\mathrm{i} \theta}+\hat{a}^{2} \mathrm{e}^{-\mathrm{i} \theta}\right)$ and then ASS (the non-classical feature) occur if

$$
Y_{\theta} \equiv \operatorname{Tr}\left[\hat{\rho}\left(\hat{Y}_{\theta}-\left\langle\hat{Y}_{\theta}\right\rangle\right)^{2}\right]-\left[\operatorname{Tr}\left(\hat{\rho} \hat{a}^{\dagger} \hat{a}\right)+\frac{1}{2}\right]<0 .
$$

Minimum value of $Y_{\theta}$ can easily be obtained and the non-classical feature can be written as [21]:

$$
Y_{\theta, \text { min }}=-\frac{1}{2}\left|\left\langle\hat{a}^{4}\right\rangle-\left\langle\hat{a}^{2}\right\rangle^{2}\right|+\frac{1}{2}\left[\left\langle\hat{a}^{\dagger 2} \hat{a}^{2}\right\rangle-\left\langle\hat{a}^{\dagger 2}\right\rangle\left\langle\hat{a}^{2}\right\rangle\right]<0
$$

For studying this non-classical feature it is customary $[18,21]$ to consider the normalized quantity $W_{\theta} \equiv Y_{\theta} /\left[\operatorname{Tr}\left(\hat{\rho} \hat{a}^{\dagger} \hat{a}\right)+\frac{1}{2}\right]$ too.

Normally-ordered characteristic function of a light beam having a Gaussian statistics may be written as

$$
\chi_{\mathrm{N}}(\zeta)=\operatorname{Tr}\left[\hat{\rho} \exp \left(\zeta \hat{a}^{\dagger}\right) \exp \left(-\zeta^{*} \hat{a}\right)\right]=\exp \left(-\left(A \zeta_{\mathrm{r}}^{2}+B \zeta_{\mathrm{i}}^{2}\right)\right),
$$

where $\zeta=\zeta_{\mathrm{r}}+\mathrm{i} \zeta_{\mathrm{i}}$. The condition that $\chi(\zeta)=\chi_{\mathrm{N}}(\zeta) \exp \left(-\frac{1}{2}|\zeta|^{2}\right) \rightarrow 0$ as $\zeta \rightarrow \infty$ [25] gives $\left(A+\frac{1}{2}\right)>0,\left(B+\frac{1}{2}\right)>0$. Also uncertainty relation $\left\langle\left(\Delta \hat{X}_{1}\right)^{2}\right\rangle\left\langle\left(\Delta \hat{X}_{2}\right)^{2}\right\rangle \geq \frac{1}{4}$ gives $(A+B+2 A B) \geq 0$. Special cases $\theta=0$ and 
$\pi / 2$ give $Y_{\theta}=Y_{1}=\frac{1}{2}\left(A^{2}+B^{2}\right)$ and $Y_{\theta}=Y_{2}=A B$, respectively. $Y_{2}$ can be amplitude-squared squeezed if one of $A$ and $B$ are negative.

Using

$$
\Gamma^{(m, n)}=\operatorname{Tr}\left(\hat{\rho} \hat{a}^{\dagger m} \hat{a}^{n}\right)=\left.(-1)^{n} \partial_{\zeta}^{m} \partial_{\zeta^{*}}^{n} \chi_{\mathrm{N}}(\zeta)\right|_{\zeta=0},
$$

we obtain

and

$$
Y_{\theta}=\frac{1}{2}(A-B)^{2} \cos \theta+A B,
$$

$Y_{\theta} /\left[\operatorname{Tr}\left(\hat{\rho} \hat{a}^{\dagger} \hat{a}\right)+\frac{1}{2}\right]=\left[(A-B)^{2} \cos \theta+2 A B\right] /(A+B+1)$.

Now let us consider the mixing of this Gaussian non-classical light beam with a coherent light beam using a Mach-Zehnder interferometer (see Fig. 1). In this scheme, we mix Gaussian light beam having annihilation operator $\hat{a}$ with a coherent light beam having annihilation operator $\hat{b}$ via beam splitter BS1, the annihilation operators $\hat{c}$ and $\hat{d}$ are output of BS1 which then is mixed via a second beam splitter BS2 with a relative phase shift $\mathrm{e}^{\mathrm{i} \delta}$.

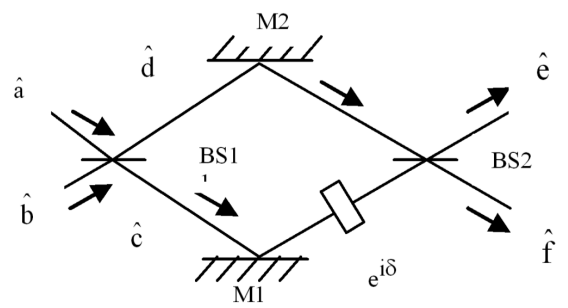

Fig. 1. Mach-Zehnder interferometer.

The different outputs can be written in terms of inputs $\hat{a}$ and $\hat{b}[21,26,27]$ as

$$
\left[\begin{array}{c}
\hat{c} \\
\hat{d}
\end{array}\right]=\left[\begin{array}{cc}
\sqrt{T_{1}} \exp \left(\mathrm{i} \phi_{1 \mathrm{~T}}\right) & \sqrt{R_{1}} \exp \left(\mathrm{i} \phi_{1 \mathrm{R}}\right) \\
-\sqrt{R_{1}} \exp \left(-\mathrm{i} \phi_{1 \mathrm{R}}\right) & \sqrt{T_{1}} \exp \left(-\mathrm{i} \phi_{1 \mathrm{~T}}\right)
\end{array}\right]\left[\begin{array}{c}
\hat{a} \\
\hat{b}
\end{array}\right],
$$

and

$$
\left[\begin{array}{c}
\hat{e} \\
\hat{f}
\end{array}\right]=\left[\begin{array}{cc}
\sqrt{T_{2}} \exp \left(\mathrm{i} \phi_{2 \mathrm{~T}}\right) & \sqrt{R_{2}} \exp \left(\mathrm{i} \phi_{2 \mathrm{R}}\right) \\
-\sqrt{R_{2}} \exp \left(-\mathrm{i} \phi_{2 \mathrm{R}}\right) & \sqrt{T_{2}} \exp \left(-\mathrm{i} \phi_{2 \mathrm{~T}}\right)
\end{array}\right]\left[\begin{array}{c}
\hat{c} \mathrm{e}^{\mathrm{i}\left(\phi_{0}+\delta\right)} \\
\hat{d} \mathrm{e}^{\mathrm{i} \phi_{0}}
\end{array}\right],
$$

where $\sqrt{R_{j}} \exp \left(-\mathrm{i} \phi_{j \mathrm{R}}\right)$ and $\sqrt{T_{j}} \exp \left(-\mathrm{i} \phi_{j \mathrm{~T}}\right)(j=1,2)$ are the coefficients for reflection and transmission for amplitudes for beam splitter BS1 or BS2, reflection coefficients for mirrors M1 or M2 are equal to $\exp \left(\mathrm{i} \phi_{0}\right)$, and beam reflected by M1 is made to experience a phase shift $\delta$.

Let us consider mixing of a Gaussian light beam (annihilation operator $\hat{a}$ ) exhibiting ASS with the coherent light beam (annihilation operator $\hat{b}$ ) of intensity as shown in Fig. 1 and straightforward calculations for minimum value of $Y_{\theta}$ for the $a$-, $c$ - and $e$-modes give 


$$
\begin{aligned}
& Y_{\theta a, \min }=A B, \operatorname{Tr}\left(\hat{\rho} \hat{a}^{\dagger} \hat{a}\right)+\frac{1}{2}=\frac{1}{2}(A+B+1) \\
& Y_{\theta c, \min }=\frac{1}{2} T_{1}^{2}(A+B)^{2}+2 R_{1} T_{1}(A+B)\left|\alpha_{0}\right|^{2}-\frac{1}{2} \mid T_{1}^{2}(B-A)^{2} \mathrm{e}^{4 \mathrm{i} \phi_{1 \mathrm{~T}}} \\
& \left.\quad+2 R_{1} T_{1}(B-A) \alpha_{0}^{2} \exp \left(2 \mathrm{i}\left(\phi_{1 \mathrm{~T}}+\phi_{1 \mathrm{R}}\right)\right)\right] \mid \\
& \operatorname{Tr}\left(\hat{\rho} \hat{c}^{\dagger} \hat{c}\right)+\frac{1}{2}=R_{1}^{2}\left|\alpha_{0}\right|^{2}+\frac{1}{2} T_{1}^{2}(A+B)+\frac{1}{2}, \\
& Y_{\theta e, \min }=\frac{1}{4}\left(\sqrt{T_{1} T_{2}}-\sqrt{R_{1} R_{2}}\right)^{4}(A+B)^{2}+\left(\sqrt{T_{1} T_{2}}-\sqrt{R_{1} R_{2}}\right)^{2} \\
& \quad \times\left(\sqrt{R_{1} T_{2}}+\sqrt{T_{1} R_{2}}\right)^{2}(A+B)\left|\alpha_{0}\right|^{2}-\frac{1}{4} \mid\left[T_{1} T_{2} \exp \left(2 \mathrm{i}\left(\alpha_{1}+\alpha_{2}+\zeta+\delta\right)\right)\right. \\
& \quad+R_{1} R_{2} \exp \left(2 \mathrm{i}\left(\beta_{2}-\beta_{1}+\delta\right)\right)-2 \sqrt{R_{1} R_{2} T_{1} T_{2}} \exp \left(\mathrm { i } \left(\alpha_{1}+\alpha_{2}+\beta_{2}\right.\right. \\
& \left.\left.\left.\quad-\beta_{1}+\delta+2 \zeta\right)\right)\right]^{2}(B-A)^{2}+2\left[T_{1} T_{2} \exp \left(2 \mathrm{i}\left(\alpha_{1}+\alpha_{2}+\zeta+\delta\right)\right)\right. \\
& \quad+R_{1} R_{2} \exp \left(2 \mathrm{i}\left(\beta_{1}-\beta_{2}+\zeta\right)\right)+2\left[T_{1} T_{2} \exp \left(2 \mathrm{i}\left(\alpha_{1}+\alpha_{2}+\zeta+\delta\right)\right)\right. \\
& \quad+R_{1} R_{2} \exp \left(2 \mathrm{i}\left(\beta_{2}-\beta_{1}+\zeta\right)\right)-2 \sqrt{R_{1} R_{2} T_{1} T_{2}} \exp \left(\mathrm { i } \left(\alpha_{1}+\alpha_{2}-\beta_{1}+\beta_{2}\right.\right. \\
& \quad+\delta+2 \zeta)]\left[R_{1} T_{2} \exp \left(2 \mathrm{i}\left(\beta_{1}+\alpha_{2}+\zeta+\delta\right)\right)+R_{2} T_{1} \exp \left(2 \mathrm{i}\left(\beta_{2}-\alpha_{1}+\zeta\right)\right)\right. \\
& \left.\quad+2 \sqrt{R_{1} R_{2} T_{1} T_{2}} \exp \left(\mathrm{i}\left(\beta_{1}+\beta_{2}-\alpha_{1}+\alpha_{2}+\delta+2 \zeta\right)\right)\right](B-A)\left|\alpha_{0}\right|^{2} \mid
\end{aligned}
$$

As the ports " $e$ " and " $f$ " are symmetrical in nature, therefore, we will study the non-classical effect for port " $e$ " and a similar discussion may occur for port " $f$ ".

\section{Discussion of the results}

With $\phi_{1 \mathrm{~T}}+\phi_{2 \mathrm{~T}}+\delta+\zeta=\phi_{2 \mathrm{R}}-\phi_{1 \mathrm{R}}+\zeta=\gamma / 2, \sigma_{1} \equiv\left(\sqrt{R_{2} T_{1}}-\sqrt{R_{1} T_{2}}\right)^{2}$, and $\sigma_{2} \equiv\left(\sqrt{T_{2} T_{1}}-\sqrt{R_{1} R_{2}}\right)^{2}$ we can discuss two cases: (i) for $\alpha_{0}=\left|\alpha_{0}\right| \exp \left(\mathrm{i}\left(\alpha_{1}-\beta_{1}\right)\right)$ and $(B-A)>0, W_{\theta e, \min }=\left(2 \sigma_{2}^{2} A B+4 \sigma_{1} \sigma_{2} A\left|\alpha_{0}\right|^{2}\right) /\left[\sigma_{2}(A+B)+2 \sigma_{1}\left|\alpha_{0}\right|^{2}+1\right]$; and (ii) for $\alpha_{0}=\left|\alpha_{0}\right| \exp \left(\mathrm{i}\left(\alpha_{1}-\beta_{1}+\pi / 2\right)\right),(A-B)>0, W_{\theta e, \min }=\left(2 \sigma_{2}^{2} A B+\right.$ $4 \sigma_{1} \sigma_{2} B\left|\alpha_{0}\right|^{2} /\left[\sigma_{2}(A+B)+2 \sigma_{1}\left|\alpha_{0}\right|^{2}+1\right]$. Taking into account these two restrictions on $A$ and $B$ and on $\alpha_{0}$, we can see that $W_{\theta a \text {,min }}<W_{\theta e \text {, min }}$ under some conditions that are dependent on the values of $T_{j}, R_{j},\left|\alpha_{0}\right|^{2}, A$, and $B$. Similar conditions may occur in the case of port " $f$ ". But, it is remarkable to note that in no way we find the situations where the maximum amount of amplitude-squared squeezing obtained by the present mixing is greater than that reported by Prakash and Mishra [21]. For example, if we maximize (i.e., most negative), $W_{a}, W_{c}$, and $W_{e}$ by a simple $\mathrm{C}++$ programming, we find that (i) $T_{1}=0.884992, A=-0.1, B=0.125,\left|\alpha_{0}\right|^{2}=25$, $W_{a}=-0.02439, W_{c}=-0.153177$; (ii) $T_{1}=0.9, T_{2}=0.6, A=-0.1, B=0.125$, $\left|\alpha_{0}\right|^{2}=25, W_{a}=-0.02439, W_{c}=-0.152802, W_{e}=-0.152776$. It is to be 
noted that Prakash and Mishra reported another non-classical features that can be enhanced by using such a procedure $[28,29]$.

\section{Ackowledgement}

Author is grateful to Prof. Hari Prakash, Prof. N. Chandra and Prof. Ranjana Prakash for their useful discussions and critical comments".

\section{References}

[1] R.J. Glauber, Phys. Rev. 131, 2766 (1963).

[2] E.C.G. Sudarshan, Phys. Rev. Lett. 10, 277 (1963).

[3] R.J. Glauber, in: Quantum Optics and Electronics, Ed. C. De Witt, Gordon and Breach, New York 1964.

[4] N. Chandra, H. Prakash, Phys. Rev. A 1, 1696 (1970).

[5] B.R. Mollow, R.J. Glauber, Phys. Rev. 160, 1076 (1967).

[6] H. Prakash, N. Chandra, Ind. J. Pure Appl. Phys. 9, 677 (1971); ibid. 9, 688 (1971); ibid. 9, 767 (1971); Lett. Nuovo Cimento 4, 1196 (1970).

[7] V.V. Dodonov, J. Opt. B, Quantum Semiclass. Opt. 4, R1 (2002).

[8] H. Takahashi, in: Advances in Communication Systems, Ed. A.V. Balakrishan, Academic Press, Orlando (FL) 1965, p. 227.

[9] Y. Yamamoto, H.A. Haus, Rev. Mod. Phys. 58, 1001 (1986).

[10] R.E. Slusher, B. Yurke, J. Lightwave Technol. 8, 466 (1990).

[11] R. Loudon, Phys. Rev. Lett. 47, 815 (1981).

[12] C.M. Caves, Phys. Rev. D 23, 1693 (1981).

[13] Quantum Optics, Experimental Gravitation, and Measurement Theory, NATO ASI Series B, Physics, Eds. P. Meystre, M.O. Scully, Plenum Press, New York 1981, Vol. 94. See articles by K.S. Thorne (p. 325), R.W.P. Drever and colleagues (p. 503), J. Hough and colleagues (p. 515).

[14] C.K. Hong, L. Mandel, Phys. Rev. A 32, 974 (1985).

[15] C.K. Hong, L. Mandel, Phys. Rev. Lett. 54, 323 (1985).

[16] M. Hillery, Opt. Commun. 62, 135 (1987).

[17] M. Hillery, Phys. Rev. A 36, 3796 (1987).

[18] C.C. Gerry, E.R. Vrscay, Phys. Rev. A 37, 1779 (1988).

[19] M.H. Mahran, A.-S. F. Obada, Phys. Rev. A 40, 4476 (1989); M.A. Mir, Phys. Rev. A 47, 4384 (1993).

[20] C.C. Gerry, S. Rodrigues, Phys. Rev. A 35, 4440 (1987); V. Buzek, I. Jex, Phys. Rev. A 41, 4079 (1990); You-bang Zhan, Phys. Lett. A 160, 498 (1991); You-bang Zhan, Phys. Rev. A 46, 686 (1992).

[21] H. Prakash, D.K. Mishra, J. Phys. B, At. Mol. Opt. Phys. 38, 665 (2005).

[22] H. Prakash, D.K. Mishra, J. Phys. B, At. Mol. Opt. Phys. 39, 2291 (2006); ibid. 40, 2531 (2007).

[23] M.T. Raiford, Phys. Rev. A 2, 1541 (1970); ibid. 9, 2060 (1974). 
[24] H. Prakash, N. Chandra, Vachaspati, Phys. Rev. A 9, 2167 (1974); Ind. J. Pure Appl. Phys. 13, 757 (1976); ibid. 13, 763 (1976); ibid. 14, 41 (1976); ibid. 14, 48 (1976).

[25] K.E. Cahill, R.J. Glauber, Phys. Rev. 177, 1882 (1969).

[26] H. Fearn, R. Loudon, Opt. Commun. 64, 485 (1987), R.A. Campos, B.E.A. Saleh, M.C. Teich, Phys. Rev. A 40, 1371 (1989); U. Leonhardt, Phys. Rev. A 48, 3265 (1993).

[27] U. Leonhardt, Measuring the Quantum State of Light, Cambridge University Press, Cambridge 1997, p. 67.

[28] H. Prakash, D.K. Mishra, Eur. Phys. J. D 45, 363 (2007). A previous version of this paper appear in: Proc. Int. Quantum Electronics Conf. 2005, p. 1299. OPAC Link: http://ieeexplore.ieee.org/servlet/opac?pnumber=10426.

[29] H. Prakash, D.K. Mishra, Opt. Spectroscopy 103, 145 (2007). 